04

\title{
Высоковольтный импульсный разряд в многофазной системе на границе раздела газ-жидкость
}

\author{
(C) А.М. Анпилов, ${ }^{1}$ Э.М. Бархударов,, И.А. Коссый, ${ }^{1}$ М.А. Мисакян,, И.В. Моряков, ${ }^{1}$ М.Г. Смирнов, ${ }^{3}$ \\ И.М. Тактакишвили ${ }^{1}$ \\ ${ }^{1}$ Институт общей фризики им. А.М. Прохорова РАН, \\ 119991 Москва, Россия \\ ${ }^{2}$ НИУ Высшая школа экономики, \\ 101000 Москва, Россия \\ ${ }^{3}$ МИРЭА - Российский технологический университет, \\ 119454 Москва, Россия \\ e-mail: igor_miw@mail.ru
}

Поступило в Редакцию 13 ноября 2020 г.

В окончательной редакции 8 декабря 2020 г.

Принято к публикации 8 декабря 2020 г.

\begin{abstract}
Приведены экспериментальные результаты исследований высоковольтного импульсного разряда, реализуемого одновременно в жидкости и газе вблизи границы раздела двух фаз. Использована многоэлектродная разрядная система с инжекцией газа в межэлектродное пространство. Предложенная схема организации разряда позволила интенсифицировать процессы обмена между жидкостью и плазмой, обрабатывать обладающую высокой проводимостью жидкость, а также пенообразующую жидкость.
\end{abstract}

Ключевые слова: искровой разряд, очистка жидкости, ультрафиолет, окислители, ударные волны.

DOI: 10.21883/JTF.2021.05.50688.311-20

\section{Введение}

Плазменно-жидкостное взаимодействие представляет собой быстро растущую междисциплинарную область исследования, включающую физику плазмы, гидродинамику, тепломассообмен, фотолиз и др. Это связано с многообразием и сложностью физико-химических процессов и возможностью решения широкого круга прикладных задач от экологии до материаловедения и здравоохранения. Результаты исследования широко представлены в работах [1-9].

Плазма в жидкости и в контакте с ней является источником сильных окислителей, например, таких, как $\mathrm{OH}, \mathrm{O}, \mathrm{H}_{2} \mathrm{O}_{2}$ и др., а также мощного УФ излучения. Плазма, по существу, является сильным окислителем, позволяющим разрушать органические и неорганические соединения в воде. Активация жидкости происходит в результате сложных физико-химических процессов, протекающих на границе раздела фаз.

Различают объемную жидкость и межфазовую область. Последняя является местом, в котором происходят наиболее важные физические и химические процессы, поэтому их интенсификация представляет значительный интерес. Проблемными вопросами плазменножидкостного взаимодействия являются также генерация импульсных разрядов в сильно проводящей жидкости $[3,10]$ и обработка пенообразующей жидкости [4]. Исследования указанных выше вопросов стимулированы, прежде всего, возможностью решения целого ряда прикладных задач. Это способствовало созданию различных разрядных систем, которые можно услов- но разделить на три типа: разряд реализуется непосредственно в жидкости, над поверхностью жидкости, включая случай, когда жидкость является электродом, и в многофазных системах (в пузырьках и струях газа в жидкости, аэрозолях, пенообразующих жидкостях и др.) [5]. Используются различные источники питания: импульсно-периодические, ВЧ, СВЧ и др. [4-7,11-14].

В настоящей работе исследуется высоковольтный импульсно-периодический разряд, который реализуется одновременно в жидкости и газе вблизи раздела фаз. Приводятся электрические и спектральные характеристики разряда, продемонстрирована возможность интенсификации процессов обмена между фазами, а также обработки сильно проводящей и пенообразующей жидкостей.

Использовалась многоэлектродная разрядная система с инжекцией газа в межэлектродное пространство $[1,13]$. Многоэлектродная система ранее исследовалась отдельно в жидкой и газовой средах. Конструкция, основные характеристики и результаты исследований представлены в $[1,14,15]$.

Ранее при исследовании многоэлектродного разряда в воде рассматривались вопросы генерации УФ излучения, $\mathrm{H}_{2} \mathrm{O}_{2}$ и $\mathrm{O}_{3}$, очистки сточной и питьевой воды от микробиологических загрязнений, конверсия метана в различные углеводороды, разрушение фенольных соединений в воде и др. [1,15].

Характеристики разрядной системы в газовой среде и информация о ее применении для решения ряда физических и прикладных задач приведены в [16]. 


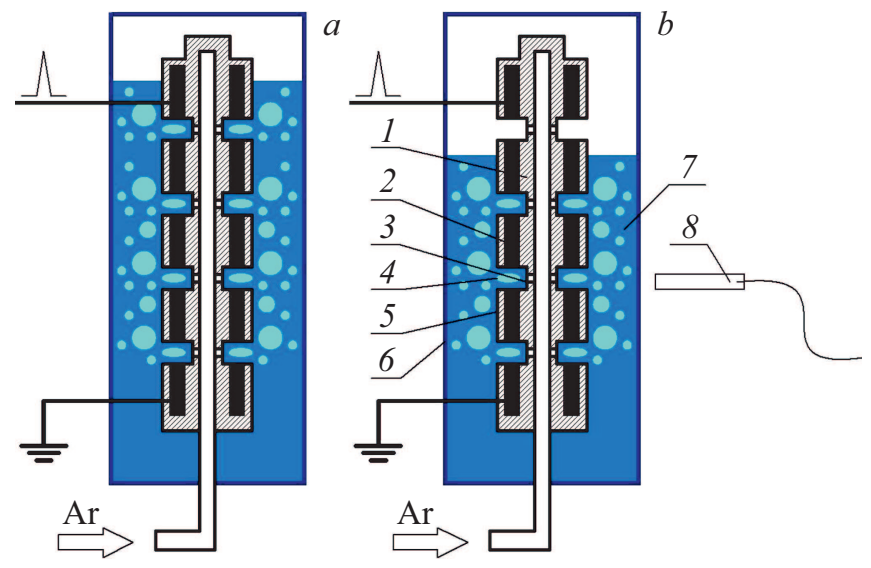

Рис. 1. Схема разрядной системы в режимах I $(a)$ и II $(b)$. 1 - диэлектрическая трубка, 2 - электроды, 3 - отверстие для подачи газа, 4 - пузырьки газа, 5 - электроизоляционное покртие электродов, $7-$ стакан из диэлектрика, $8-$ зонд для спектральных измерений.

\section{1. Экспериментальные результаты и обсуждения}

На рис. 1 приведена схема экспериментальной установки и два варианта ее использования: $a-$ разрядник целиком погружен в воду (режим I), $b-$ один из разрядных промежутков находится над поверхностью воды (режим II). Конструкция разрядного устройства: на внешней поверхности диэлектрической трубки из фторопласта 1 , охватывая ее, располагаются цилиндрические электроды 2 из нержавеющей стали с одинаковым зазором $1.5 \mathrm{~mm}$ (в ряде экспериментов вместо нержавеющей стали использовались титан и медь). Диаметр трубки $d_{t}=12 \mathrm{~mm}$. В стенках трубки в межэлектродном пространстве имеются отверстия 3 диаметром $d_{0} \sim 0.8 \mathrm{~mm}$, через которые из полости трубки в воду поступают пузыри газа 4. Боковая поверхность электродов покрыта изоляционным материалом 5. Камеpa 6 , изготовленная из прозрачного пластика, объемом $V=250 \mathrm{~cm}^{3}$ частично заполнялась водой 7. Спектральные измерения проводились при помощи зонда 8 . Воздух над поверхностью жидкости находился при атмосферном давлении. Число разрядных промежутков равнялось четырем. В качестве газа, инжектируемого в воду, использовался аргон. Его расход составлял $\leq 21 / \mathrm{min}$. Проводимость воды менялась от 5 до $1.5 \cdot 10^{4} \mu \mathrm{S} / \mathrm{cm}$. При измерении проводимости использовался кондуктометр HANNA DIST-5 HI 98312.

Разряд формировался с помощью генератора высоковольтных импульсов с энергией накопительного конденсатора $E \leq 1.6 \mathrm{~J}$, частотой следования импульсов $f \leq 100 \mathrm{~Hz}$. Амплитуда тока и напряжения составляли $\sim 250-300 \mathrm{~A}$ и $\sim 20 \mathrm{kV}$ соответственно.

Принципиальная схема разрядной цепи приведена на рис. 2, где $C_{c}=4 \cdot 10^{-10} \mathrm{~F}$ и $L_{c}=10^{-6} \mathrm{H}, R_{0}=6 \cdot 10^{3} \Omega$, $C_{0}=8 \cdot 10^{-9} \mathrm{~F}, L_{0}=3 \cdot 10^{-5} \mathrm{H}$, Thyratron - тиратрон
ТГИ1-1000/25, $R_{c}-$ сопротивление разрядного промежутка. Для измерения напряжения и тока использовались делитель напряжения 1:1000 (Tektronix P6015А) 1 и пояс Роговского $1: 10$ (Stangenes Industries) 2.

Интерес к исследованиям по формированию импульсных разрядов в проводящей жидкости связан с возможностью их использования в экологии и биомедицине, в частности, в устройствах для стерилизации воды и жидких аэрозолей [3,17-20]. Решение этой задачи связано с определенными трудностями. Для облегчения инициации разряда используются различные методы, в том числе и инжекция пузырей газа в жидкость $[10]$. В $[1,14]$ данный метод был использован в многоэлектродной разрядной системе. Дополнительно улучшить условия формирования разряда можно за счет размещения, по крайней мере, одного разрядного промежутка в газовой фазе.

Для высоковольтного импульсного разряда длительностью $\sim 1 \mu$ s требуется достаточно высокая крутизна нарастания импульса напряжения. Это связано с тем, что из-за высокой проводимости жидкости до достижения пробойного напряжения значительная часть энергии импульса затрачивается на нагрев жидкости. Предлагаемая схема организации разряда позволяет значительно нивелировать этот эффект. При подаче высоковольтного импульса все электроды, находящиеся в воде, имеют потенциал земли. Следовательно, напряжение прикладывается между электродами, находящимися в газовой среде. По мере нарастания импульса напряжения происходит пробой воздушного промежутка и практически все напряжение оказывается приложенным к электродам, находящимся в воде. Таким образом, разряд в воде на начальной стадии формируется при более высоком напряжении. Данный процесс можно регулировать за счет изменения длины воздушного разрядного промежутка.

На рис. 3 представлены осциллограммы тока и напряжения для режимов работы I и II, где 1 и $2-$ времена предпробойной и канальной стадий искрового разряда соответственно.

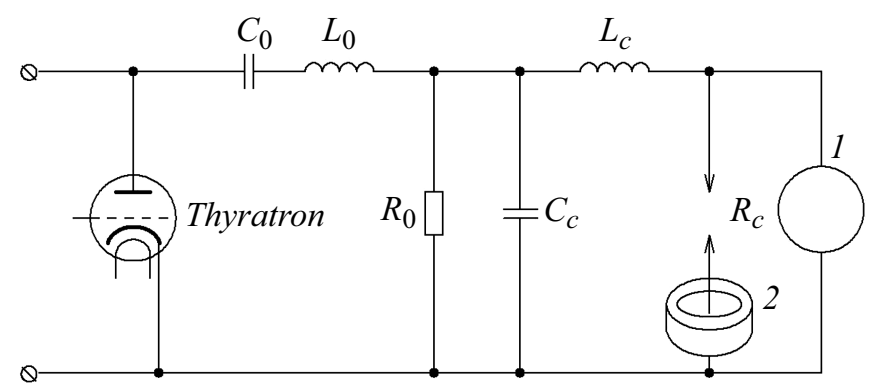

Рис. 2. Принципиальная схема разрядной цепи. $C_{c}-$ емкость кабеля, $L_{c}-$ индуктивность кабеля, $R_{0}-$ внутреннее сопротивление генератора, $C_{0}$ - емкость накопительного конденсатора, $L_{0}$ - индуктивность генератора, $R_{c}$ - сопротивление разрядного промежутка, Thyratron - тиратрон ТГИ1-1000/25, 1 - делитель напряжения, 2 - пояс Роговского. 

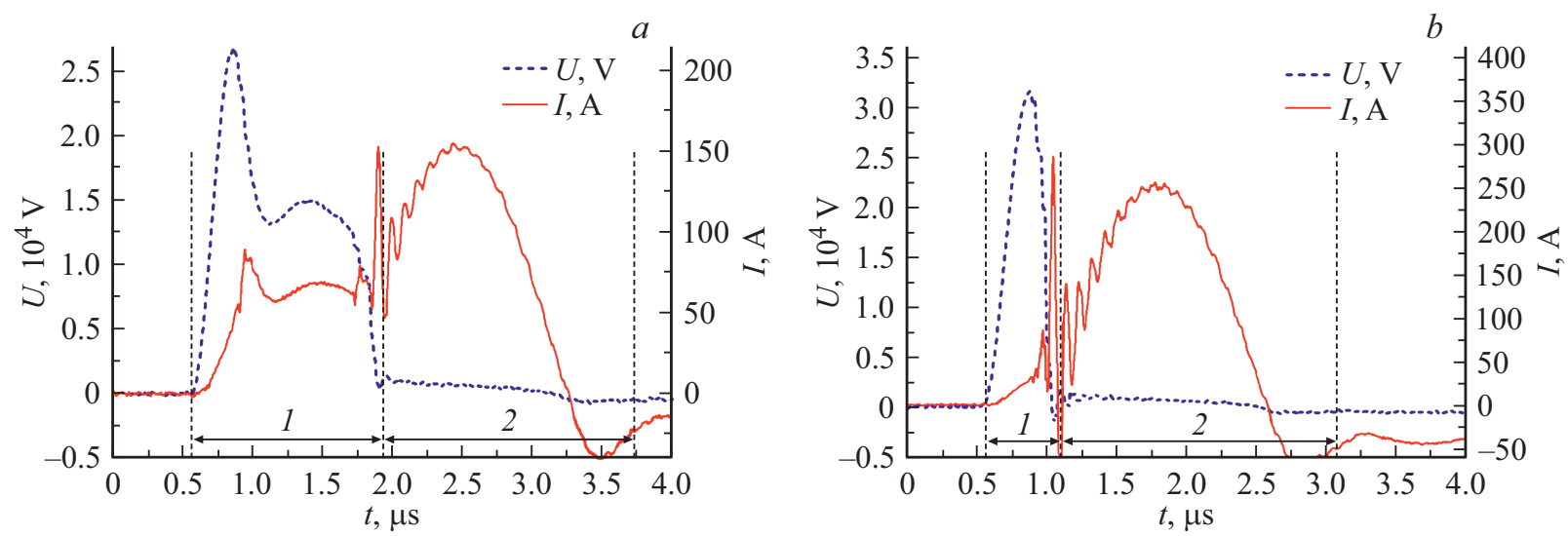

Рис. 3. Осциллограммы тока и напряжения для режима $I(a)$ и II $(b)$, проводимость воды $\sigma=400 \mu \mathrm{S} / \mathrm{cm}$.
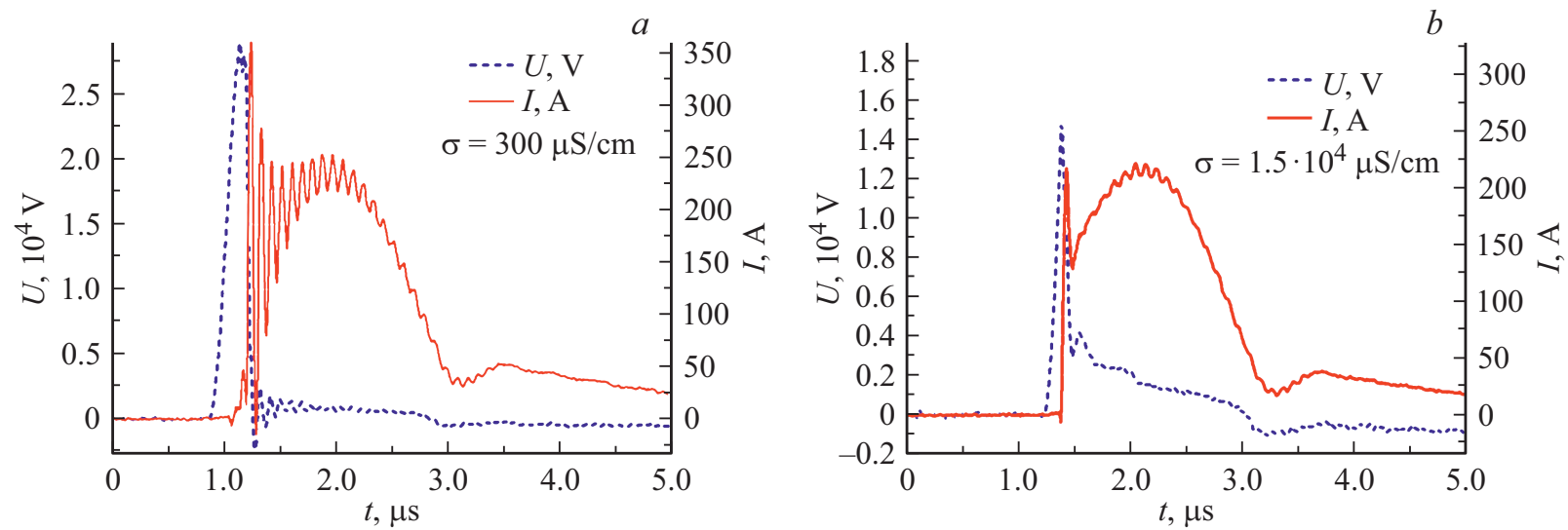

Рис. 4. Осциллограммы тока и напряжения $\sigma, \mu \mathrm{S} / \mathrm{cm}: a-300, b-1.5 \cdot 10^{4}$.
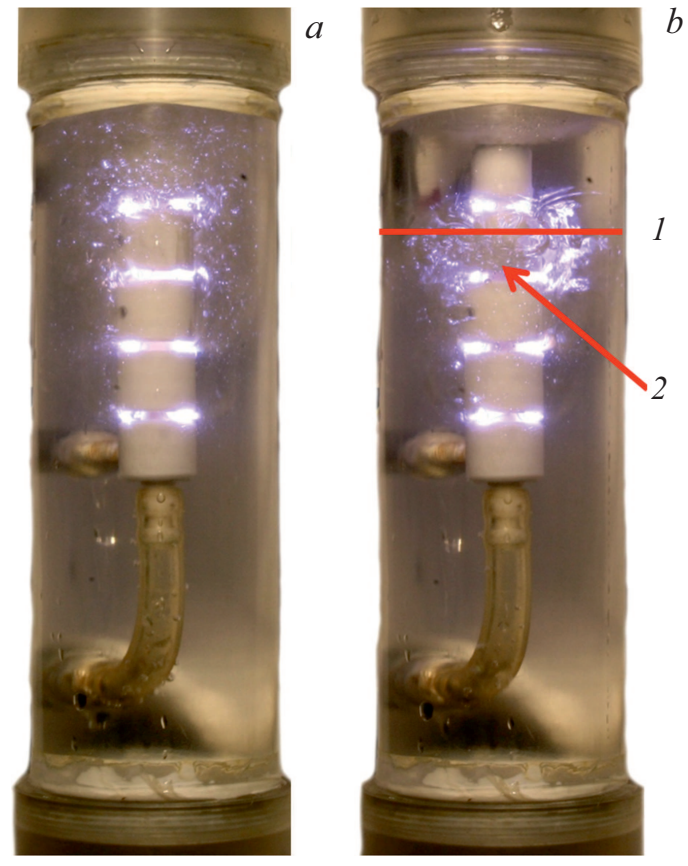

Pис. 5. Фотографии разряда в режимах I $(a)$ и II $(b), 1-$ граница раздела жидкости и газа, 2 - зона образования многофазной системы.
Из сравнения осциллограмм рис. 3 следует, что наличие разрядного промежутка в газе приводит к существенному уменьшению длительности предпробойной стадии разряда. Согласно оценкам, сделанным на основе приведенных осциллограмм, в режиме I в предпробойную стадию вкладывается $0.5 \mathrm{~J}$, а в канальную $0.1 \mathrm{~J}$. В режиме II практически вся энергия расходуется в канальной стадии.

Наличие разрядного промежутка в газовой фазе (режим II) позволяет генерировать разряд в широком интервале изменения проводимости воды. Иллюстрацией этого являются осциллограммы тока и напряжения (рис. 4), полученные при различной проводимости воды $\sigma_{1}=300 \mathrm{muS} / \mathrm{cm}$ и $\sigma_{2}=1.5 \cdot 10^{4} \mu \mathrm{S} / \mathrm{cm}$ (морская вода). Характер осциллограмм практически совпадает. Основная энергия выделяется в канальной стадии разряда.

На рис. 5 представлены фотографии разряда в режимах I и II. Горизонтальная линия 1 на рис. $5, b$ соответствует границе раздела двух фаз вода-воздух.

О характере разряда в газе и в пузырьках газа в воде можно судить по спектрам излучения, представленным на рис. 6 , где $a$ соответствует разряду в пузырьках газа в жидкости и $b-$ разряду в газе. Спектрограммы получены с помощью спектрографа S2000 Ocean Optics. Диапазон регистрации прибора 250-800 nm, спектраль- 

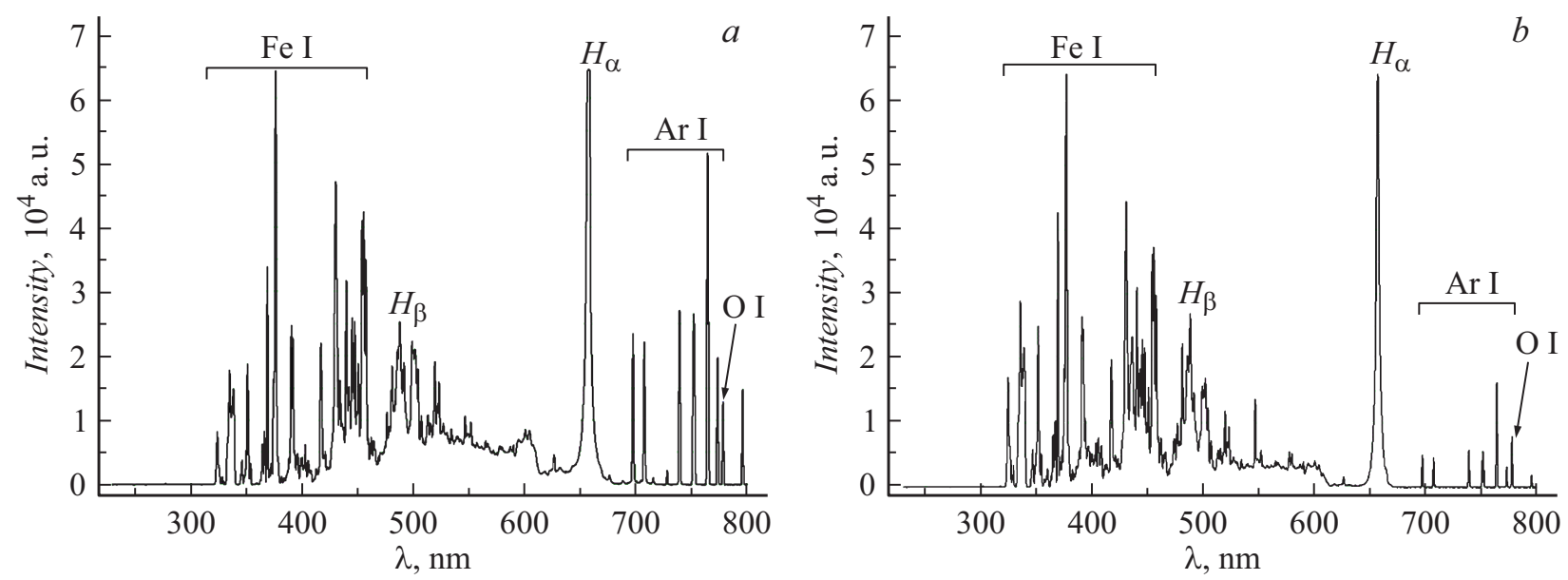

Рис. 6. Интегральные спектры излучения: $a-$ в пузырьках газа в жидкости, $b-$ разряд в газе.
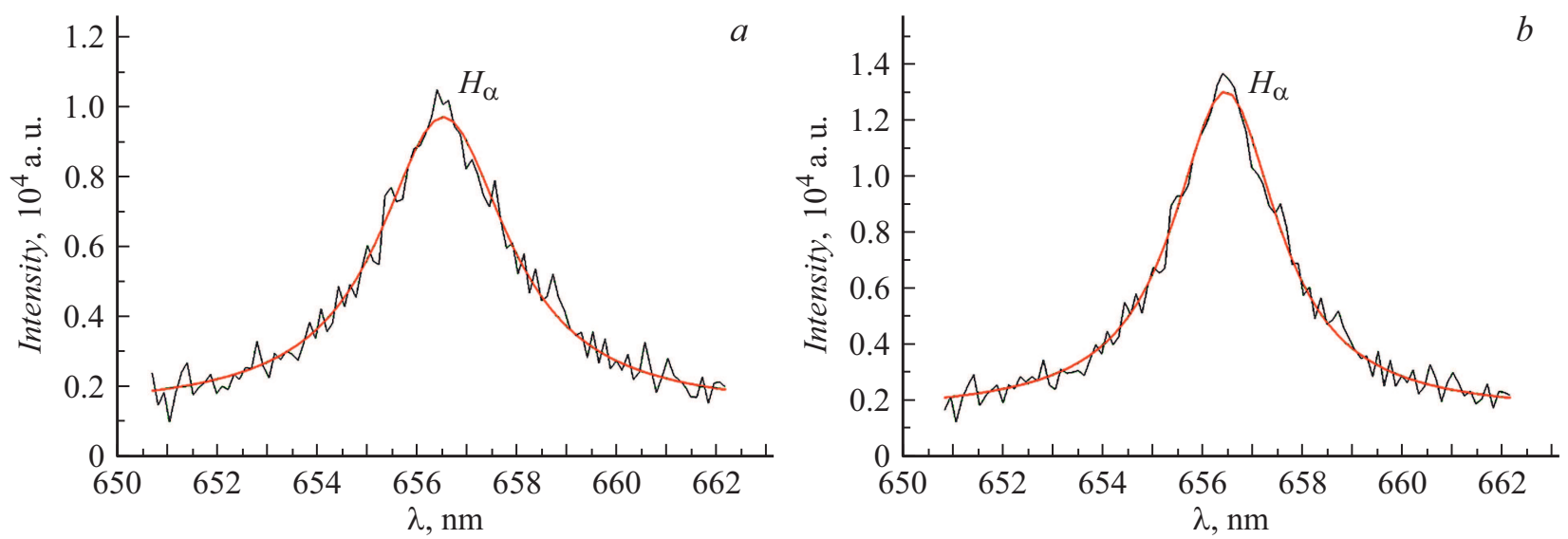

Рис. 7. Контур линии $H_{a}: a-$ под водой, в пузырьках газа, $b-$ над водой.

ное разрешение $\sim 1 \mathrm{~nm}$. Стенка камеры (рис. 1,7 ) прозрачна для излучения с длиной волны > $300 \mathrm{~nm}$. Спектры получены при частоте следования импульсов $f=10 \mathrm{~Hz}$, время регистрации $t=2 \mathrm{~s}$. Измерение спектров проводилось при помощи зонда (рис. 1,8 ), диаметр световода $600 \mu \mathrm{m}$, полоса пропускания от $180 \mathrm{~nm}$. Калиброванный зонд позволял регистрировать излучение только из одного разрядного промежутка.

Из полученных результатов следует, что спектры излучения, соответствующие жидкой и газовой фазам, практически совпадают. В спектрах выделяются линии аргона, железа $\mathrm{Fe}(\mathrm{I})(300-460 \mathrm{~nm})$, триплет кислорода $(777 \mathrm{~nm})$ и линии водорода $\mathrm{H}_{\alpha}$ и $\mathrm{H}_{\beta}$. Отсутствие азота в спектре (рис. 6, $b$ ) свидетельствует о том, что разряд над поверхностью воды протекает практически в атмосфере аргона. Соответствующие профили линии $\mathrm{H}_{\alpha}$ приведены на рис. 7. По их уширению, связанному с эффектом Штарка в электрическом поле плазмы, определена концентрация электронов в пузырьках газа в воде (рис. 7,a) и в газе над поверхностью воды (рис. $7, b)$. Полученные данные практически совпадают $-n_{e} \approx 10^{17} \mathrm{~cm}^{-3}[14,16,21]$.
Приведенные результаты позволяют утверждать, что рассматриваемый нами разряд представляет собой совокупность микроплазменных образований, для которых характерен высокий удельный энерговклад. Действительно, при общей длине всех разрядных промежутков $L=6 \mathrm{~mm}$, диаметре искрового канала $d \leq(2-3) \cdot 10^{-2} \mathrm{~cm}$ и энерговкладе в искровой канал $E=0.6 \mathrm{~J}$, удельный энерговклад составляет $\geq 1 \mathrm{~kJ} / \mathrm{cm}^{3}$.

Кроме того, можно считать, что разряд над поверхностью воды и в пузырьках газа в жидкости протекает по схожему сценарию.

Рассмотрим характерные особенности плазменножидкостного взаимодействия, реализуемого в описанных выше экспериментах.

Ранее нами было показано, что многоэлектродный разряд в воде с инжекцией газа развивается по принципу поверхностного разряда [16] вдоль границы раздела жидкость-газ. Вопросы развития разряда в газовых полостях и пузырьках рассмотрены в ряде работ. В частности, в [22] экспериментально показано, что искровой разряд в газовой полости в воде развивается по ее поверхности. Согласно [23], стримерный разряд 


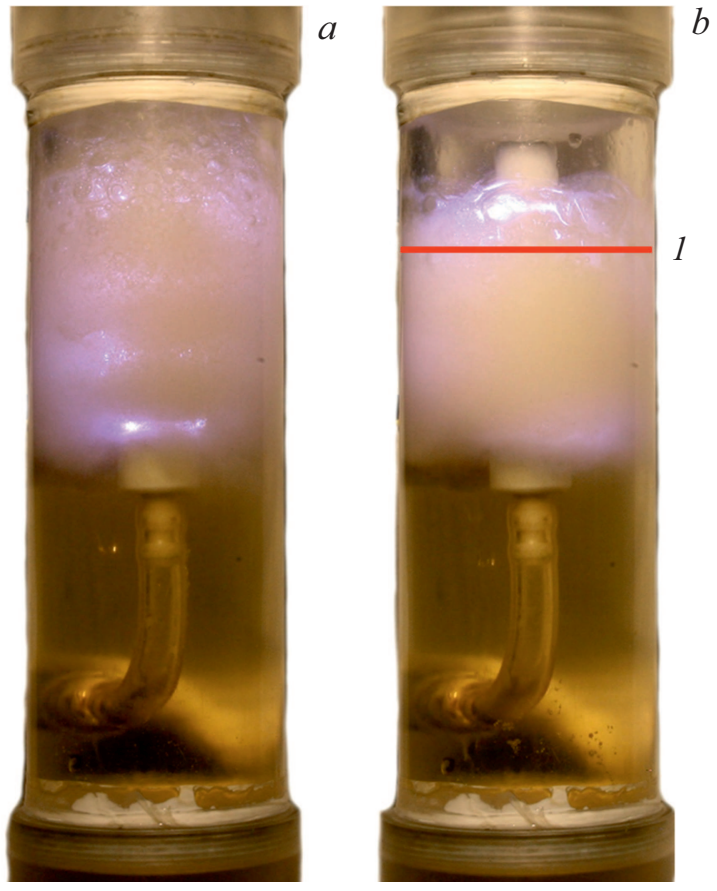

Рис. 8. Фотографии разряда в пенообразующей жидкости в режимах I $(a)$ и II $(b), 1$ - граница раздела газ-жидкость.

в пузырьках газа в воде развивается вдоль поверхности жидкости. В [24] с помощью компьютерного моделирования показано, что разряд в пузырьке газа, помещенного в жидкость с высокой диэлектрической проницаемостью, развивается вдоль ее поверхности. Подробно вопросы развития разряда в пузырьках газа в жидкости рассмотрены в ряде работ, в частности [7,25-28].

Микроплазменные образования в воде, помимо непосредственного воздействия на воду, генерируют сильные окислители (OH, O, $\mathrm{H}_{2} \mathrm{O}_{2}, \mathrm{O}_{3}$ и др.) и являются источником мощного УФ излучения и сильных акустических волн. Возможно также развитие кавитационных процессов. Отметим, что газовый пузырь, инжектируемый в жидкость, распадается на множество мелких пузырей, что увеличивает эффективность воздействия сильных окислителей на жидкость.

Вследствие формирования разряда в жидкости и газе у границы раздела фаз возникает многофазовая область (газ, жидкость, плазма, аэрозоль, пары воды), в которой происходят интенсивные процессы обмена. Свой вклад в эти процессы вносит барботация жидкости, и при достаточно высоком энерговкладе гидродинамический эффект, подобный подводному взрыву у границы жидкости [29].

Для газовой среды у границы раздела фаз, помимо таких факторов, как непосредственное действие плазмы разряда, генерации сильных окислителей, УФ излучения, характерным является генерация ударных волн.

Из приведенных результатов следует, что плазменножидкостное взаимодействие в предлагаемой разрядной системе является многоканальным: одновременно реализуются различные физические и плазмохимические процессы, что может способствовать повышению эффективности плазможидкостного взаимодействия при решении различных прикладных задач.

Как отмечалось выше, одной из проблем плазменножидкостного взаимодействия является обработка пенообразующей жидкости [4]. Обильное выделение пены существенно снижает эффективность воздействия разряда на жидкость в результате выноса жидкости из зоны разряда и в некоторых случаях приводит к гашению разряда.

В режиме II разряд в газе генерирует ударную волну, которая способна предотвратить выход пены из жидкости. Это подтверждается фотографиями, приведенными на рис. 8 , где $a$ - режим I и $b-$ режим II. Из фотографии следует, что в режиме II выход пены из жидкости предотвращен. В качестве жидкости использовалось пиво.

Данный метод был опробован в лабораторных условиях при обработке отходов пивной промышленности с целью уменьшения биологической и химической кислородной активности жидкости.

\section{Заключение}

Исследован высоковольтный импульсный разряд, характерной особенностью которого является генерация плазмы одновременно в газе и жидкости у границы раздела.

Полученные результаты указывают на то, что разряд в газе и в пузырьках газа в жидкости развивается по схожему сценарию и представляет собой микроплазменные образования с удельным энерговкладом $\geq 1 \mathrm{~kJ} / \mathrm{cm}^{3}$ и концентрацией электронов $\sim 10^{17} \mathrm{~cm}^{-3}$. Образовавшаяся на границе газ-жидкость многофазная область приводит к интенсификации процессов обмена между фазами. С учетом процессов, протекающих в объеме жидкости, можно считать, что плазможидкостное взаимодействие в рассмотренной системе является сложным и многоканальным, включающим в себя различные физические и плазмохимические процессы. Последнее может приводить к синергетическим эффектам.

Наличие разрядного промежутка в газовой фазе позволяет обрабатывать воду с высокой проводимостью, вПлоть до $\sigma_{2}=1.5 \cdot 10^{4} \mu \mathrm{S} / \mathrm{cm}$, что важно при решении большого числа прикладных задач, например, таких, как обработка производственных отходов, в биомедицине обработка физиологических растворов, стерилизация воды и жидких аэрозолей, в гидроакустике генерация ударных волн в воде.

Предлагаемая система позволяет также решить востребованную технологическую задачу - обработку пенообразующей жидкости за счет генерации ударных волн в газовой фазе у поверхности жидкости. 
В зависимости от конкретной прикладной задачи параметры разрядной системы могут легко меняться: энерговклад, инжектируемый газ, число микроплазменных образований и др.

Продемонстрированные в работе принципы обработки жидкости могут лечь в основу физико-химических исследований плазменно-жидкостного взаимодействия, а также решения широкого круга прикладных задач.

\section{Конфликт интересов}

Авторы заявляют, что у них нет конфликта интересов.

\section{Список литературы}

[1] A.M. Anpilov, E.M. Barkhudarov, Yu.B. Bark, Yu.V. Zadiraka, M. Christofi, Yu.N. Kozlov, I.A. Kossyi, V.A. Kop'ev, V.P. Silakov, M.I. Taktakishvili, S.M. Temchin. J. Phys. D, 34 (6), 993 (2001). DOI: 10.1088/0022-3727/34/6/322

[2] B.R. Locke, M. Sato, P. Sunka, M.R. Hoffmann, J.-S. Chang. Ind. Eng. Chem. Res., 45 (3), 882 (2006). DOI: $10.1021 / \mathrm{ie} 050981 \mathrm{u}$

[3] P. Bruggeman, C. Leys. J. Phys. D: Appl. Phys., 42, 1 (2009). DOI: $10.1088 / 0022-3727 / 42 / 5 / 053001$

[4] S. Samukawa, M. Hori, S. Rauf, K. Tachibana, P. Bruggeman, G. Kroesen, J.C. Whitehead, A.B. Murphy, A.F. Gutsol, S. Starikovskaia, U. Kortshagen, J.-P. Boeuf, T.J. Sommerer, M.J. Kushner, U. Czarnetzki, N. Mason. J. Phys. D: Appl. Phys., 45, 1 (2012). DOI: 10.1088/0022-3727/45/25/253001

[5] P.J. Bruggeman, M.J. Kushner, B.R. Locke, J.G.E. Gardeniers, W.G. Graham, D.B. Graves, R.C.H.M. Hofman-Caris, D. Maric, J.P. Reid, E. Ceriani, D.F. Rivas, J.E. Foster, S.C. Garrick, Y. Gorbanev, S. Hamaguchi, F. Iza, H. Jablonowski, E. Klimova, J. Kolb, F. Krcma, P. Lukes, Z. Machala, I. Marinov, D. Mariotti, S.M. Thagard, D. Minakata, E.C. Neyts, J. Pawlat, Z.L. Petrovic, R. Pflieger, S. Reuter, D.C. Schram, S. Schröter, M. Shiraiwa, B. Tarabová, P.A. Tsai, J.R.R. Verlet, T. von Woedtke, K.R. Wilson, K. Yasui, G. Zvereva. Plasma Sources Sci. Technol., 25, 1 (2016). DOI: $10.1088 / 0963-0252 / 25 / 5 / 053002$

[6] Ю.А. Лебедев. Физика плазмы, 43 (6), 685 (2017). DOI: $10.7868 / \mathrm{S} 0367292117060105$

[7] N.Yu. Babaeva, R.S. Berry, G.V. Najdis, B.M. Smirnov, É.E. Son, D.V. Tereshonok. TVT, 54 (5), 792 (2016). DOI: $10.7868 / \mathrm{S} 0040364416050057$

[8] A. Hamdan, J.-L. Liu, M.S. Cha. Plasma Chem. Plasma Process., 38, 1003 (2018). DOI: 10.1007/s11090-018-9918-y

[9] V.I. Goryachev, F.G. Rutberg, V.N. Fedyukovich. Appl. Energy, 36, 35 (1998).

[10] К.А. Наугольных, Н.А. Рой. Электрические разряды в воде (Наука, М., 1971)

[11] Yu.S. Akishev, M.E. Grushin, V.B. Karal'nik, A.E. Monich, M.V. Pan'kin, N.I. Trushkin. Plasma Physics Reports, 32 (12), 1052 (2006). Original Russian Text published in Fizika Plazmy, 32 (12), 1142 2006). DOI: $10.1134 / \mathrm{S} 1063780 \mathrm{X} 06120087$

[12] Y. Akishev, M. Grushin, V. Karalnik, N. Trushkin. Pure Appl. Chem., 80 (9), 1953 (2008). DOI: 10.1351/pac200880091953

[13] А.М. Анпилов, Э.М. Бархударов, Н.К. Бережецкая, С.И. Грицинин, А.М. Давыдов, Ю.Н. Козлов, И.А. Коссый, М.А. Мисакян, С.М. Темчин, В.Г. Ральченко, П.А. Гущин, Е.В. Иванов. ЖТФ, 81 (11), 48 (2011).

DOI: $10.1134 / \mathrm{S} 1063784211110028$
[14] E.M. Barkhudarov, I.A. Kossyi, Yu.N. Kozlov, S.M. Temchin, M.I. Taktakishvili, N. Christofi. J. Atom. Molecular Phys., 2013, Article ID 429189, 12 p. (2013). DOI: $10.1155 / 2013 / 429189$

[15] A.M. Anpilov, E.M. Barkhudarov, N. Christofi, V.A. Kop'ev, I.A. Kossyi, M.I. Taktakishvili, Yu.V. Zadiraka. J. Water and Health, 2 (4), 267 (2004). DOI: 10.2166/wh.2004.0024

[16] A.M. Anpilov, E.M. Barkhudarov, Yu.N. Kozlov, I.A. Kossyi, M.A. Misakyan, I.A. Moryakov, M.I. Taktakishvili, N.M. Tarasova, S.M. Temchin. Plasma Phys. Rep., 45 (3), 246 (2019). DOI: $10.1134 /$ S1063780X19020016

[17] Yu.S. Akishev, M.E. Grushin, V.B. Karal'nik, A.E. Monich, M.V. Pan'kin, N.I. Trushkin, V.P. Kholodenko, V.A. Chugunov, N.A. Zhirkova, I.A. Irkhina, E.N. Kobzev. Plasma Phys. Rep., 32, 1052 (2006). DOI: 10.1134/S1063780X06120087

[18] M.W. Ahmed, S. Choi, K. Lyakhov, U. Shaislamov, R.K. Mongre, D.K. Jeong, R. Suresh, H.R. Lee. Plasma. Phys. Rep., 23, 381 (2017). DOI: 10.1134/S1063780X17030011

[19] Y.C. Hong, H.J. Park, B.J. Lee, W.S. Kang, H.S. Uhm. Phys. Plasmas, 17, 053502 (2010). DOI: 10.1063/1.3418371

[20] V. Kolikov, P. Rutberg. Pulsed Electrical Discharges for Medicine and Biology (Springer, 2015), ISBN 978-3-31918129-5

[21] Г. Грим. Уширение спектральных линий в плазме (Мир, M., 1978)

[22] В.М. Шмелев, А.М. Анпилов, Э.М. Бархударов. Прикладная физика, 5, 55 (2005).

[23] P. Bruggeman, C. Leys, J. Vierendeels. J. Phys. D: Appl. Phys., 40, 1937 (2007).

[24] N.Yu. Babaeva, A.N. Bhoj, M.J. Kushner. J. Phys. D: Appl. Phys., 42, 132003 (5p.) (2009). DOI: $10.1088 / 0022-3727 / 42 / 13 / 132003$

[25] Yu.S. Akishev, G.I. Aponin, M.E. Grushin, V.B. Karalnik, A.V. Petryakov, N.I. Trushkin. J. Optoelectron. Adv. Mater., 10, 1917 (2008).

[26] Yu. Akishev, F. Arefi-Khonsari, A. Demir, M. Grushin, V. Karalnik, A. Petryakov, N. Trushkin. Plasma Sources Sci. Technol., 24, 065021 (15p.) (2015). DOI: $10.1088 / 0963-0252 / 24 / 6 / 065021$

[27] Yu. Hayashi, N. Takada, H. Kanda, M. Goto. Plasma Sources Sci. Technol., 24, 1 (2015). DOI: $10.1088 / 0963-0252 / 24 / 5 / 055023$

[28] J.E. Foster, B. Sommers, S. Gucker. Jpn. J. Appl. Phys., 54 (1S), 01AF05 (2015). DOI: $10.7567 /$ JJAP.54.01AF05

[29] С.Г. Андреев, А.В. Бабкин, Ф.А. Баум, Н.А. Имховик, И.Ф. Кобылкин, В.И. Колпаков, С.В. Ладов, В.А. Одинцов, Л.П. Орленко, В.Н. Охитин, В.В. Селиванов, В.С. Соловьев, К.П. Станюкович, В.П. Челышев, Б.И. Шехтер. Физика взрыва, под ред. Л.П. Орленко. Изд. 3-е, перераб. (Физматлит, М., 2002) 